\title{
Quantitative Analysis and Efficient Surface Modification of Silica Nanoparticles
}

\author{
Hak-Sung Jung, Doo-Sik Moon, and Jin-Kyu Lee \\ Department of Chemistry, Seoul National University, Seoul 151-747, Republic of Korea \\ Correspondence should be addressed to Jin-Kyu Lee, jinklee@snu.ac.kr \\ Received 31 January 2012; Accepted 8 April 2012 \\ Academic Editor: Hongchen Chen Gu
}

Copyright () 2012 Hak-Sung Jung et al. This is an open access article distributed under the Creative Commons Attribution License, which permits unrestricted use, distribution, and reproduction in any medium, provided the original work is properly cited.

\begin{abstract}
Aminofunctional trialkoxysilanes such as aminopropyltrimethoxysilane (APTMS) and (3-trimethoxysilylpropyl)diethylenetriamine (DETAS) were employed as a surface modification molecule for generating monolayer modification on the surface of silica $\left(\mathrm{SiO}_{2}\right)$ nanoparticles. We were able to quantitatively analyze the number of amine functional groups on the modified $\mathrm{SiO}_{2}$ nanoparticles by acid-base back titration method and determine the effective number of amine functional groups for the successive chemical reaction by absorption measurements after treating with fluorescent rhodamine B isothiocyanate (RITC) molecules. The numbers of amine sites measured by back titration were 2.7 and $7.7 \mathrm{ea} / \mathrm{nm}^{2}$ for $\mathrm{SiO}_{2}$-APTMS and $\mathrm{SiO}_{2}$-DETAS, respectively, while the numbers of effective amine sites measured by absorption calibration were about one fifth of the total amine sites, namely, 0.44 and $1.3 \mathrm{ea} / \mathrm{nm}^{2}$ for $\mathrm{SiO}_{2}$-APTMS(RITC) and $\mathrm{SiO}_{2}$-DETAS(RITC), respectively. Furthermore, it was confirmed that the reactivity of amino groups on the surface-modified silica nanoparticles could be maintained in ethanol for more than 1.5 months without showing any significant differences in the reactivity.
\end{abstract}

\section{Introduction}

Since Werner Stöber has developed the synthetic method for preparing silica particles, colloidal silica particles have been intensively investigated to understand the reaction mechanism and control the size and size uniformity [1], and they have been used in many areas such as silicon wafer polishing, beverage clarification, and composite materials [2]. Recently, silica nanoparticles were represented as one of the most widespread nanomaterials in use because they have several features: (i) ease of preparation through hydrolysiscondensation reaction from relatively low-priced precursor molecules such as tetraethyl orthosilicate (TEOS) in the presence of acid or base catalysts, (ii) possible surface modification with various organosilicon compounds, and (iii) its biocompatibility without showing acute toxicity [3-5].

The silanol groups, $\mathrm{Si}-\mathrm{OH}$, on the silica surface can be easily modified to various functional groups by treating with organotrialkoxysilane $\left(\mathrm{RSi}\left(\mathrm{OR}^{\prime}\right)_{3}\right)$ compounds or methallylsilanes together with catalyst [6], and surface modification of the silica nanoparticles with biorecognition molecules can make a specific interaction with receptor sites of living systems. Based on this surface modification technique, there has been a great amount of research effort to use the silica nanoparticles as carriers for drug or gene deliveries [7-10]. Due to its stability and good biocompatibility along with easy surface modification, silica was also used as a surface coating material for many nanomaterials [11]. Organotrialkoxysilanes produce silanol groups by hydrolysis, which can be condensed with surface $\mathrm{Si}-\mathrm{OH}$ group to form stable siloxane bond, Si-O-Si, for surface modification. However, they can also engage in self-condensation to form gel or oily oligomers to be precipitated within few hours in the presence of base catalysts. Therefore, there is a dilemma whether to use the large excess amount of organotrialkoxysilane to maximize the coverage of $\mathrm{SiO}_{2}$ surface or to use minimum amount to prevent the formation of any unwanted self-condensed side products. These self-condensed side products, in practice, are usually removed by repeating centrifugation/redispersion processes because the actual size of self-condensation products are very small compared to the size of $\mathrm{SiO}_{2}$ nanoparticles. Therefore, there is always a possibility that these selfcondensed products further condense with $\mathrm{Si}-\mathrm{OH}$ groups of the silica surface, resulting in a thick coating layer instead of 
generating a monolayer modification. Even though the thick coating layer of organotrialkoxysilane has the advantage, in some cases, of giving more reactive functional groups for reactions, precise control of the number of surface functional groups cannot be achieved in a reproducible and reliable manner.

Among the various organotrialkoxysilane molecules for the modification of silica surfaces, 3-aminopropyltrimethoxysilane (APTMS) and 3-aminopropyltriethoxysilane (APTES) were widely explored by many researchers. The aminofunctional groups are known to enhance the dispersibility and miscibility of silica fibers [12], and to be used as a linkage unit to attach other functional molecules [13]. The surface modification of silica has been investigated in bulk surfaces and micron size silica beads by many characterization techniques [14-19]. A reaction mechanism was suggested as through the interaction between the amino group of APTES and surface $\mathrm{Si}-\mathrm{OH}$ group in anhydrous condition $[14,20-22]$ or through the self catalytic effect of the amino group of APTES in polar alcoholic condition [23]. Interestingly, it was known that aminofunctional trialkoxysilanes such as APTMS and APTES are readily soluble in water to give solutions of unlimited stability at their natural $\mathrm{pH}$ in which normal organotrialkoxysilanes cause rapid condensation of $\mathrm{Si}-\mathrm{OH}$ groups to form insoluble gels, and the internal hydrogen bonding was suggested to explain their lack of reactivity [12].

In this study, we have investigated the characteristic feature of aminofunctional ligand molecules such as APTMS and DETAS in ethanol solution, forming monolayer on the surface of $\mathrm{SiO}_{2}$ nanoparticles instead of generating a thick coating layer. The total number of amino groups on the surface of $\mathrm{SiO}_{2}$ nanoparticle was quantitatively analyzed by a simple acid-base back titration method and the effective number of amino groups for the successive chemical reactions was also determined by spectroscopic measurements after treating with fluorescent Rhodamine B isothiocyanate (RITC) molecule. It was also confirmed that the number of amino groups on the surface-modified silica nanoparticles, and their reactivity could be maintained in ethanol at room temperature for more than 1.5 months without showing any significant differences.

\section{Experimental}

2.1. Materials. 3-Aminopropyltrimethoxysilane (APTMS), 3-aminopropyltriethoxysilane (APTES), and (3-trimethoxysilylpropyl)diethylenetriamine (DETAS) were purchased from Gelest. Tetraethylorthosilicate (TEOS) was purchased from TCI. Rhodamine B isothiocyanate (RITC), methyl acrylate (MA), and ethylenediamine (EDA) were purchased from Aldrich. Ammonia solution was purchased from Samchun. All organic solvents were used as received without any further purification.

The size and shape of silica nanoparticles were characterized by transmission electron microscope (TEM; Carl Zeiss, LIBRA 120) and field emission scanning electron microscope (FE-SEM; Hitachi, S-4300), dynamic light scattering spectrophotometer (DLS; Qudix, scatteroscope I).
Fourier transform infrared (FT-IR) spectra were recorded using a JASCO FT/IR-600 Plus spectrometer for wavelengths ranging from 400 to $4000 \mathrm{~cm}^{-1}$ to study the surface of silica nanoparticles. The absorption spectra of surface modified silica nanoparticles decorated with RITC were measured by UV-visible spectrometer (Sinco, S-3100).

2.1.1. Synthesis of Silica Nanoparticles. To a TEOS $(2.5 \mathrm{~mL})$ solution in $115 \mathrm{~mL}$ of dried ethanol, $3.75 \mathrm{~mL}$ of aqueous ammonium hydroxide solution $(14.6 \mathrm{M})$ and $3.75 \mathrm{~mL}$ of water were added while stirring. After $12 \mathrm{~h}$ of stirring, silica nanoparticles were isolated by centrifugation at a speed of 15,000 rpm and the supernatant was removed. The isolated products were redispersed in ethanol. The washing process with centrifugation/redispersion was repeated 3 times. Finally, the redispersed nanoparticle solution was centrifuged at a speed of 2,000 rpm to remove any aggregated particles. The purified $\mathrm{SiO}_{2}$ nanoparticles were homogeneously dispersed in ethanol. The size and shape of the nanoparticles were characterized by TEM, FE-SEM, and DLS.

\subsubsection{Surface Modification of Silica Nanoparticles with Amino-} functional Trimethoxysilanes. $10 \mathrm{~mL}$ of $\mathrm{SiO}_{2}$ nanoparticle solution ( $5 \mathrm{mg} / \mathrm{mL}$ of ethanol) was added into ten different vials. Appropriate amounts of APTMS (or DEATS) were added into each vial to maintain the conditions of surface modification; weight ratios of $\mathrm{SiO}_{2}$ : APTMS (or DETAS) were varied from $1: 0.01$ to $1: 0.1$. After $12 \mathrm{hr}$ of stirring at room temperature, modified silica nanoparticles were isolated and purified by centrifugation/redispersion processes (for $10 \mathrm{~min}$ at $15,000 \mathrm{rpm}, 3$ times) to remove the excess APTMS (or DETAS). Finally, the purified $\mathrm{SiO}_{2}$-APTMS (or $\mathrm{SiO}_{2}$-DETAS) nanoparticles were kept dispersed in ethanol.

2.1.3. Quantification of the Number of Amine Sites on the Modified $\mathrm{SiO}_{2}$ Nanoparticles by Back Titration. $10 \mathrm{mg}$ of modified silica nanoparticles was dispersed in $20 \mathrm{~mL}$ of $1.0 \mathrm{mM} \mathrm{HCl}$ solution and stirred for $30 \mathrm{~min}$. Nanoparticles was separated by centrifugation at $15,000 \mathrm{rpm}$ for $10 \mathrm{~min}$, and $10 \mathrm{~mL}$ of supernatant was collected to be titrated with the standardized $1.0 \mathrm{mM} \mathrm{NaOH}$ solution in the presence of phenolphthalein indicator. From the difference of $\mathrm{HCl}$ concentration after treating with modified $\mathrm{SiO}_{2}$ nanoparticles, the molar concentration of amine sites on the modified $\mathrm{SiO}_{2}$ nanoparticles $(10 \mathrm{mg})$ was calculated. This value was converted into the number of amine sites per unit area $\left(\mathrm{nm}^{2}\right)$ of the surface of $\mathrm{SiO}_{2}$ nanoparticle based on the density of bulk silica $(2.2 \mathrm{~g} / \mathrm{mL})$ and the surface area of $100 \mathrm{~nm} \mathrm{SiO} 2$ nanoparticle.

2.1.4. Quantification of the Number of Amine Sites on the Modified $\mathrm{SiO}_{2}$ Nanoparticles by Absorption Measurement after Coupling with RITC. $10 \mathrm{~mL}$ of the dispersed $\mathrm{SiO}_{2}$-APTMS (or $\mathrm{SiO}_{2}$-DETAS) nanoparticles in ethanol $(5 \mathrm{mg} / \mathrm{mL}$ solution), prepared from various ratios of $\mathrm{SiO}_{2}$ : APTMS (or DETAS), was treated with RITC $\left(34.4 \mathrm{mg}, 6.42 \times 10^{-2} \mathrm{mmol}\right)$, and the mixed solution was stirred for $12 \mathrm{hr}$ at $\mathrm{RT}$. $\mathrm{SiO}_{2}-$ APTMS(RITC) $\left\{\right.$ or $\mathrm{SiO}_{2}$-DETAS(RITC) $\}$ nanoparticles were 


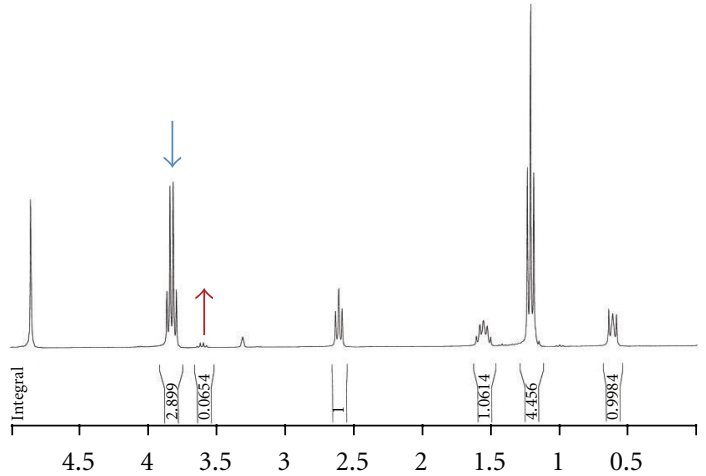

(a)

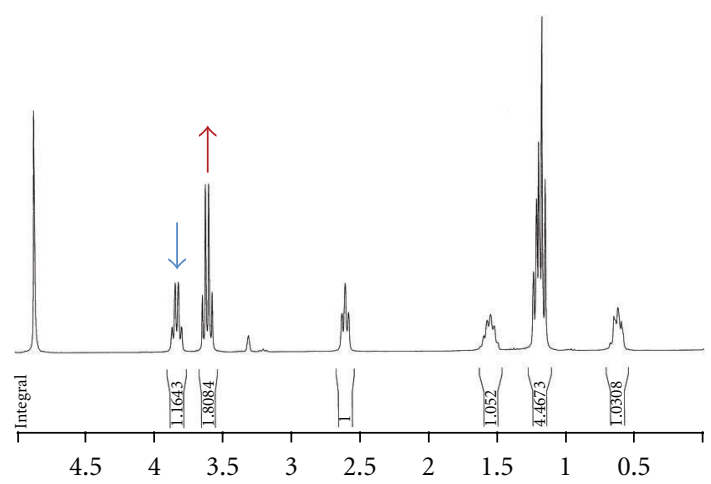

(c)

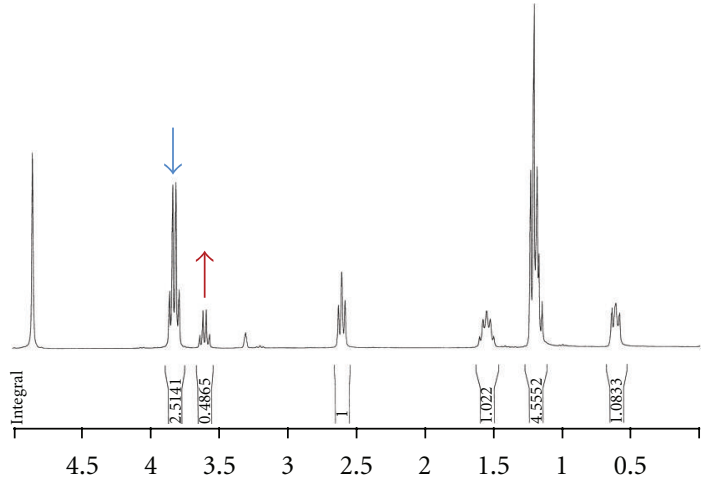

(b)

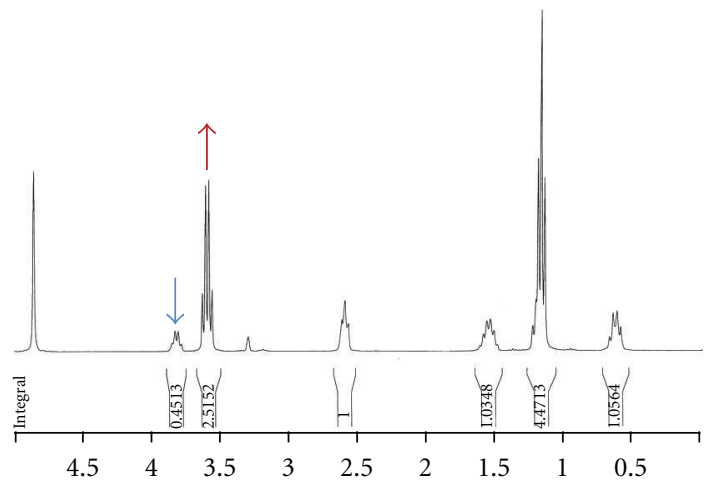

(d)

Figure 1: ${ }^{1} \mathrm{H}-\mathrm{NMR}$ spectra of APTES in $\mathrm{CD}_{3} \mathrm{OD}$ after (a) just mixing, (b) $30 \mathrm{~min}$, (c) $3 \mathrm{~h}$, and (d) $6 \mathrm{~h}$.

isolated by centrifugation at a speed of $15,000 \mathrm{rpm}$ and repeatedly washed by centrifugation and redispersion until no RITC was detected from the supernatant. All the purified $\mathrm{SiO}_{2}$-APTMS(RITC) \{or $\mathrm{SiO}_{2}$-DETAS(RITC) $\}$ nanoparticles, prepared from different ratios of $\mathrm{SiO}_{2}$ : APTMS (or DETAS), were redispersed in $10 \mathrm{~mL}$ of ethanol. Small portions of each solution of modified nanoparticles $(0.5 \mathrm{~mL}$ of $\mathrm{SiO}_{2}$-APTMS(RITC) or $0.3 \mathrm{~mL}$ of $\mathrm{SiO}_{2}$-DETAS(RITC), resp.) were diluted into $5 \mathrm{~mL}$ of ethanol for the absorption measurements. The absorptions of each sample were measured by UV-Vis spectrophotometry. A series of diluted solutions of RITC in ethanol was prepared to make the calibration curve and the absorption values were also measured by UV-Vis spectrophotometry.

2.1.5. Reaction of the Surface Amine Sites of $\mathrm{SiO}_{2}-A P T M S$ by a Process Analogous to Dendrimer Synthesis. $50 \mathrm{mg}$ of $\mathrm{SiO}_{2}$-APTMS nanoparticles was stirred with methyl acrylate $(\mathrm{MA} ; 1.8 \mathrm{~mL})$ in $10 \mathrm{~mL}$ of methanol in a vial at $25^{\circ} \mathrm{C}$, with hydroquinone as an inhibitor. After $48 \mathrm{hr}$ of stirring, the modified silica nanoparticles were purified by centrifugation/redispersion in methanol (for $10 \mathrm{~min}$ at 15,000 rpm, 3 times) to remove the excess $\mathrm{MA}$. The purified $\mathrm{SiO}_{2}-\mathrm{MA}$ nanoparticles were dispersed in methanol and treated with ethylenediamine (EDA; $5.01 \mathrm{~mL}$, concentration $7.5 \mathrm{M}$ ) at $25^{\circ} \mathrm{C}$. After $48 \mathrm{hr}$ of stirring, the $\mathrm{SiO}_{2}$-EDA nanoparticles were purified by centrifugation/redispersion in methanol (for $10 \mathrm{~min}$ at 15,000 rpm, 3 times), and the purified $\mathrm{SiO}_{2}$ EDA nanoparticles were kept dispersed in methanol.

2.1.6. Long Term Stability Test of Aminofunctional Groups on $\mathrm{SiO}_{2}$-DETAS by Coupling with RITC. $10 \mathrm{~mL}$ of the dispersed $\mathrm{SiO}_{2}$-DETAS nanoparticles in ethanol $(5 \mathrm{mg} / \mathrm{mL}$ solution), after storing for 1.5 months, was treated with RITC (34.4 mg, $6.42 \times 10^{-2} \mathrm{mmol}$ ) and the mixed solution was stirred for $12 \mathrm{hr}$ at RT. $\mathrm{SiO}_{2}$-DETAS(RITC) nanoparticles were isolated by centrifugation at a speed of $15,000 \mathrm{rpm}$ and repeatedly washed by centrifugation and redispersion until no RITC was detected from the supernatant. The purified $\mathrm{SiO}_{2}$ DETAS(RITC) nanoparticles were redispersed in $10 \mathrm{~mL}$ of ethanol. $0.3 \mathrm{~mL}$ of $\mathrm{SiO}_{2}$-DETAS(RITC) nanoparticles were diluted into $5 \mathrm{~mL}$ of ethanol for the absorption measurements. The absorption of $\mathrm{SiO}_{2}$-DETAS(RITC) was measured by UV-Vis spectrophotometry.

\section{Results and Discussion}

To check the stability of aminofunctional trialkoxysilanes in aqueous and alcoholic solutions as reported in the literature [12], APTES was dissolved in deuterated methanol $\left(\mathrm{CD}_{3} \mathrm{OD}\right)$ and the changes were monitored by ${ }^{1} \mathrm{H}-\mathrm{NMR}$. As shown in Figure 1, new methylene peaks next to oxygen atom were increased at $3.6 \mathrm{ppm}$ with time, corresponding to free ethanol molecule resulting from either alcohol exchange 


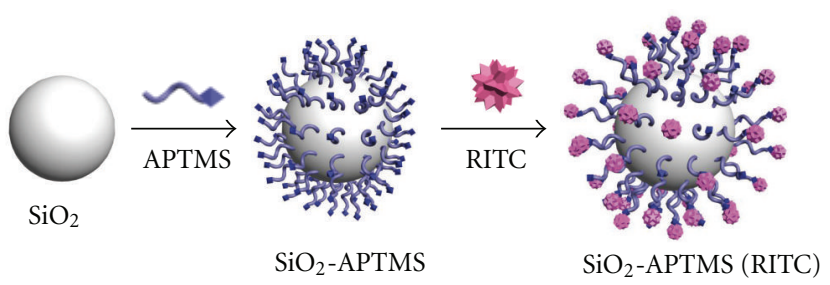

(a)

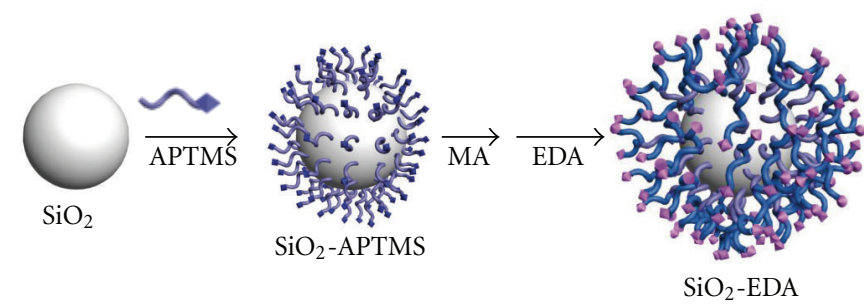

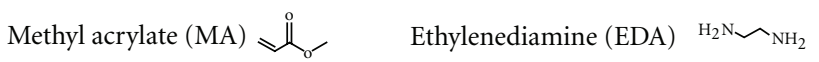

(b)

Scheme 1: Illustration of the surface modification of silica nanoparticle by APTMS followed by treatment with (a) RITC to measure the absorption and (b) methyl acrylate and ethylenediamine to increase the number of amine sites.

with methanol solvent or hydrolysis with small amount of water in the system. Even though almost all the ethoxy groups were replaced from APTES after $6 \mathrm{~h}$, the clear solution remained without forming an insoluble gel by condensation reaction. A similar but much faster hydrolysis reaction was observed in $\mathrm{D}_{2} \mathrm{O}$ solution (see Figure $\mathrm{S} 1$ in supplementary materials available online at doi:10.1155/2012/593471), and again no gel was formed. When the concentration of APTES was increased as high as $0.1 \mathrm{M}$ where silica nanoparticles were usually prepared in the presence of basic $\mathrm{NH}_{4} \mathrm{OH}$ catalyst, a clear solution was maintained for several weeks. The methanol solution of APTES was also checked by electrospray ionization mass spectrometry (ESI-MS). The very slow alcohol exchange and hydrolysis reaction was confirmed again and no higher molecular ion peaks (higher than 300) were detected after $6 \mathrm{~h}$ as shown in Figure S2.

Based on the results of stability tests of APTES in alcoholic solution confirming no self-condensation of APTES in a meaningful speed, the surface modification of silica nanoparticles having an average size of $100 \pm 15 \mathrm{~nm}$ was carried out with aminopropyltrimethoxysilane (APTMS) and (3-trimethoxysilylpropyl)diethylenetriamine (DETAS) in ethanol solution (Scheme 1(a)). Silica nanoparticles were prepared by the known method after slight modifications $[1,12]$, and dispersed in ethanol $(5 \mathrm{mg} / \mathrm{mL}$ of $\mathrm{EtOH})$. $10 \mathrm{~mL}$ of this solution was treated with different amounts of APTMS in the range of 1 to $10 \mathrm{wt} \%$. After the mixed solution was stirred for $12 \mathrm{~h}$, the modified silica nanoparticle, $\mathrm{SiO}_{2}$-APTMS, was isolated and washed by centrifugation 3 times and redispersion in ethanol. To easily check the number of effective amine groups on the surface of silica naoparticle, these modified silica nanoparticles were reacted with a large excess amount (more than $10^{3}$ times excess compared to the amounts of used APTMS) of Rhodamine B isothiocyanate (RITC) to attach fluorescent rhodamine units by forming thiourea linkage, which has become one of the useful bioconjugation methods [24]. $\mathrm{SiO}_{2}$-APTMS(RITC) nanoparticles were again purified by centrifugation and redispersion in ethanol until no fluorescence from free RITC was detected in the supernatant. The absorption intensities from $\mathrm{SiO}_{2}$-APTMS(RITC) nanoparticles clearly showed that the absorption from chemically attached RITC units had increased as a function of the amount of APTMS used for the surface modification and seemed to reach the saturation point at mixing ratio of around $1: 0.05\left(\mathrm{SiO}_{2}\right.$ : APTMS, wt ratio) (Figure 2(a)). A very similar trend was observed from the modification experiments with DETAS, even though the absorption intensities were much higher due to the multiple amine sites (Figure 2(b) and Figure S3). These results showing saturation in absorption intensity seem to imply the formation of monolayer of APTMS and DETAS instead of the generation of a thick coating layer in our modification condition.

To confirm the formation of monolayer modification, silica nanoparticles before and after the surface modifications were precisely characterized by transmission electron microscopy (TEM) and dynamic light scattering (DLS) spectrophotometer. As shown in Figures 3(a)-3(c), TEM images of $\mathrm{SiO}_{2}, \mathrm{SiO}_{2}$-APTMS, and $\mathrm{SiO}_{2}$-DETAS did not show any significant size differences after the surface modifications. However, the size measured by DLS clearly showed the increment of the hydrodynamic sizes of $\mathrm{SiO}_{2}$ nanoparticles in water from 137 to 141 , and finally to $169 \mathrm{~nm}$ (Figures $3(\mathrm{~d})-3(\mathrm{f}))$; the size from DLS is usually reported to be bigger 

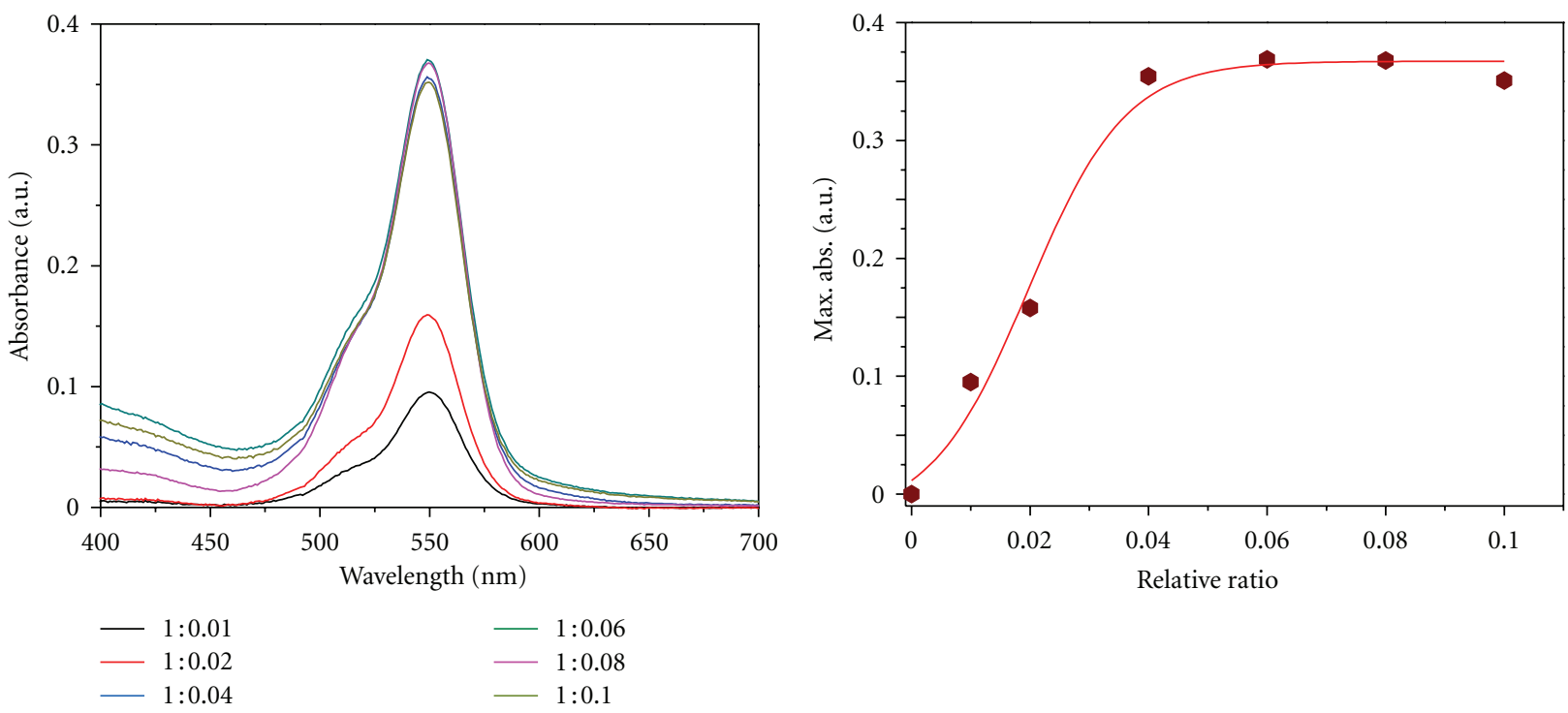

(a)

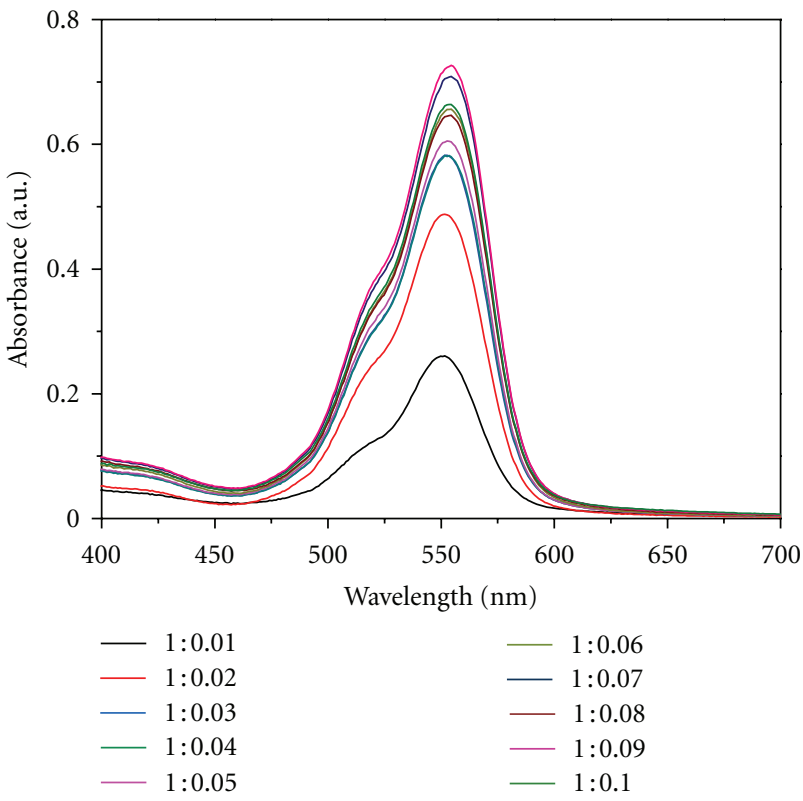

(b)

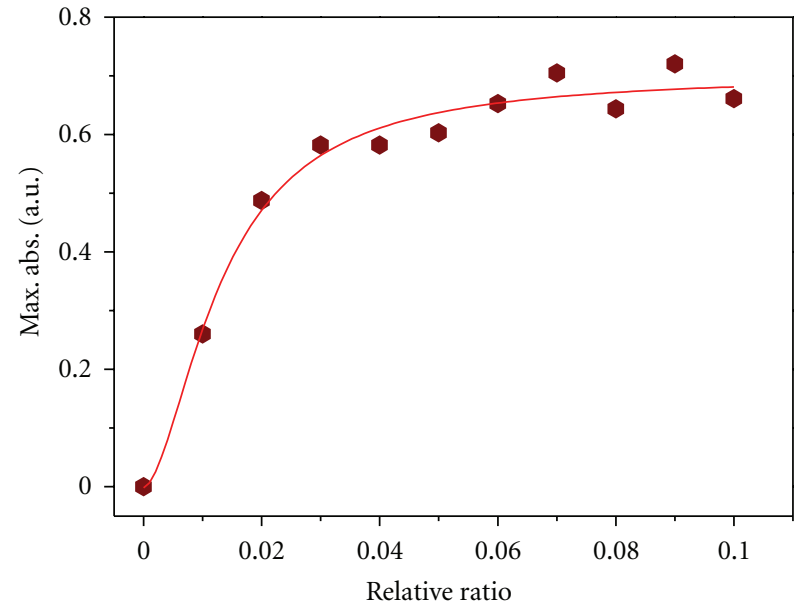

(c)

(d)

FIGURE 2: The absorption spectra of (a) $\mathrm{SiO}_{2}$-APTMS(RITC) and (c) $\mathrm{SiO}_{2}$-DETAS(RITC) nanoparticles prepared with various amounts of surface modification ligands, (b) and (d) relative intensity plots for each case clearly showing the absorption from chemically attached RITC units was increased as a function of the amount of APTMS used for the surface modification and seemed to reach the saturation point at around the mixing ratio of $1: 0.05 .0 .5 \mathrm{~mL}$ of $\mathrm{SiO}_{2}$-APTMS(RITC) or $0.3 \mathrm{~mL}$ of $\mathrm{SiO}_{2}$-DETAS(RITC) was diluted into $5 \mathrm{~mL}$ of ethanol for the absorption measurements.

than that from TEM due to the surrounded water molecules and swelling effect of surface molecules. A comparison of FTIR spectra of $\mathrm{SiO}_{2}$-DETAS with $\mathrm{SiO}_{2}$ clearly showed that the functional groups from DETAS molecule were observed after the surface modification (Figure 4).

Since the surface-to-volume ratio of nanoparticles is dramatically increased compared to bulk materials, the actual number of aminofunctional groups in the modified silica samples would be reasonably high enough to be directly measured by simple acid-base titration. Due to the feature of amino group as a weak base and the possible aggregation and scattering problems during the titration, back titration method was employed for the direct measurement of amino groups on the surface of silica nanoparticles. Back titration is a technique designed to resolve problems when analytes are in nonsoluble solid phase having either too weak or too slow characteristics to give a valid reaction; the unknown concentration of a base sample is determined by reacting it with 


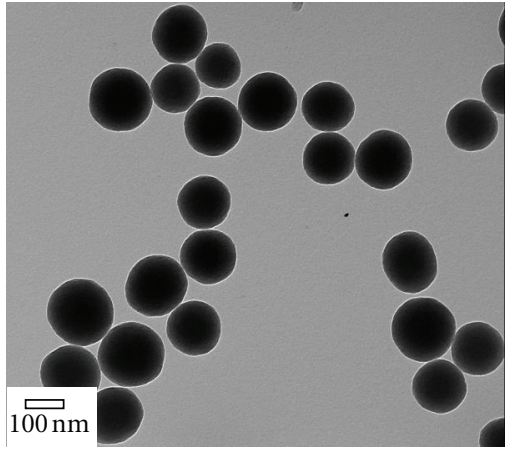

(a)

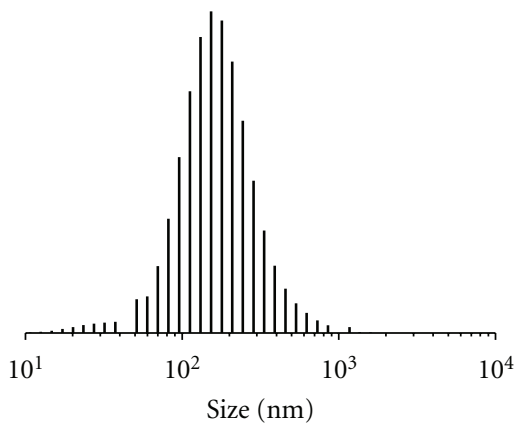

(d)

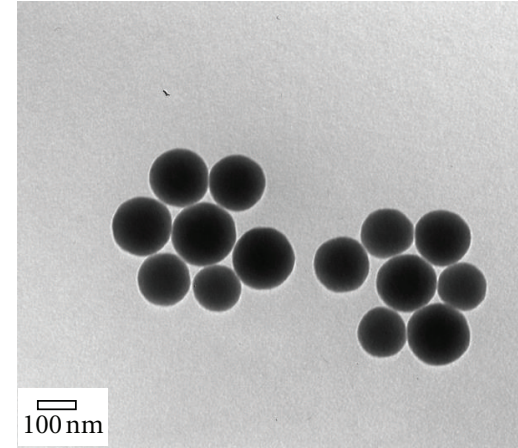

(b)

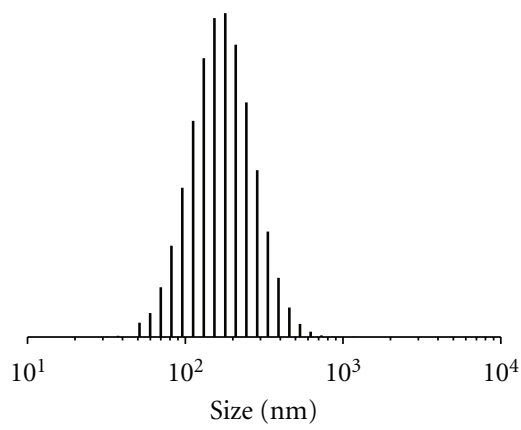

(e)

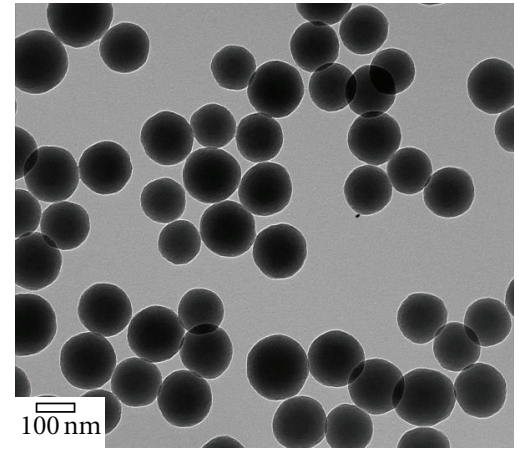

(c)

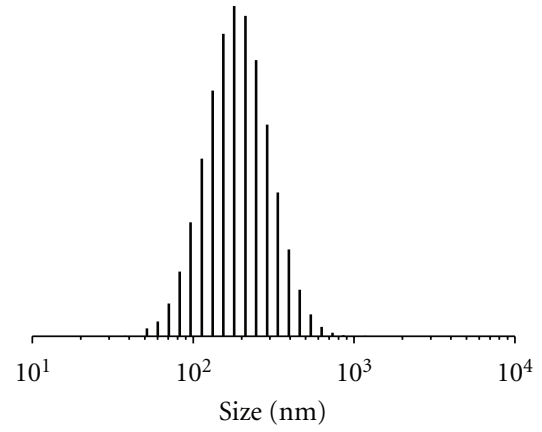

(f)

FIgure 3: TEM images of (a) $\mathrm{SiO}_{2}$, (b) $\mathrm{SiO}_{2}$-APTMS, and (c) $\mathrm{SiO}_{2}$-DETAS nanoparticles, and size distributions measured by DLS for (d) $\mathrm{SiO}_{2}(137 \mathrm{~nm}),(\mathrm{e}) \mathrm{SiO}_{2}$-APTMS $(141 \mathrm{~nm})$, and (f) $\mathrm{SiO}_{2}$-DETAS $(169 \mathrm{~nm})$ nanoparticles.

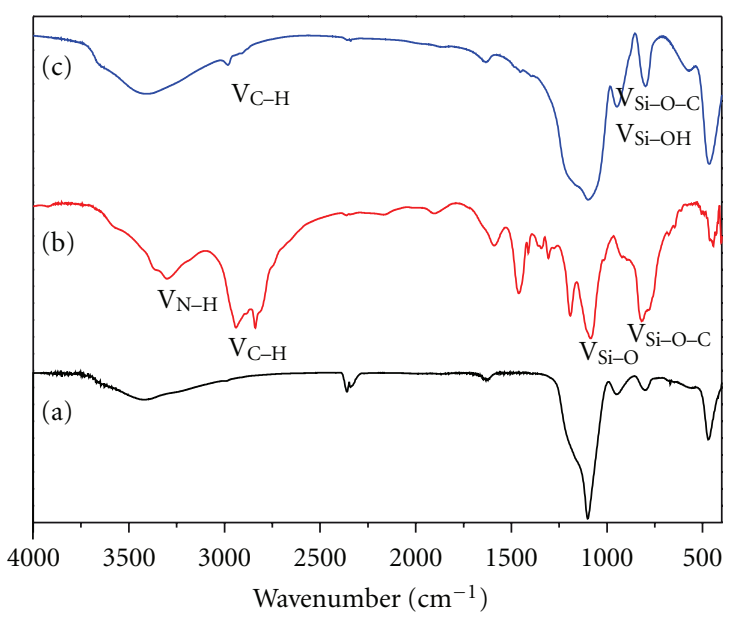

Figure 4: FT-IR spectra of (a) $\mathrm{SiO}_{2}$ nanoparticle, (b) DETAS ligand molecule, and (c) $\mathrm{SiO}_{2}$-DETAS nanoparticle.

an excess volume of acid with a known concentration and then titrating the resulting mixture with the standardized base solution to give the differences of the acid concentration which corresponds to the concentration of unknown base sample [25]. $10 \mathrm{mg}$ of modified silica nanoparticles was dispersed in $20 \mathrm{~mL}$ of $0.01 \mathrm{M} \mathrm{HCl}$ solution and stirred for $30 \mathrm{~min}$. Nanoparticles were separated by centrifugation at $15,000 \mathrm{rpm}$ for $10 \mathrm{~min}$, and $10 \mathrm{~mL}$ of supernatant was
TABle 1: Measured numbers of silanol and amine sites on the various silica surfaces from references as well as this work.

\begin{tabular}{|c|c|c|c|c|}
\hline & Type of silica & $\begin{array}{l}\text { Number } \\
\text { of silanol } \\
\text { sites } \\
\left(\mathrm{ea} / \mathrm{nm}^{2}\right)\end{array}$ & $\begin{array}{l}\text { Number of } \\
\text { amine sites } \\
\left(\mathrm{ea} / \mathrm{nm}^{2}\right)\end{array}$ & Method (reference) \\
\hline (1) & Aerosol & 4.8 & 1.6 & IR [26] \\
\hline (2) & Fumed silica & 7 & 2.3 & $\begin{array}{c}\text { Inverse reaction } \\
\text { chromatography } \\
{[27]}\end{array}$ \\
\hline (3) & Colloidal silica & $3.3 \sim 6.6$ & $1.2 \sim 2.2$ & IR [28] \\
\hline (4) & $\mathrm{SiO}_{2}$-APTMS & & $2.7 \pm 0.35$ & Titration \\
\hline (5) & $\mathrm{SiO}_{2}$-DETAS & & $7.7 \pm 0.19$ & " \\
\hline (6) & $\mathrm{SiO}_{2}-\mathrm{EDA}$ & & $6.8 \pm 1.10$ & " \\
\hline (7) & $\mathrm{SiO}_{2}$-APTMS & & $0.44 \pm 0.01$ & Absorption \\
\hline (8) & $\mathrm{SiO}_{2}$-DETAS & & $1.3 \pm 0.08$ & $"$ \\
\hline (9) & $\mathrm{SiO}_{2}$-EDA & & $0.99 \pm 0.07$ & " \\
\hline
\end{tabular}

collected to be titrated with the standardized $0.01 \mathrm{M} \mathrm{NaOH}$ solution in the presence of phenolphthalein indicator (see Table S1 for the summary of titration results and calculations).

Table 1 summarizes the number of amine sites measured by the titration as well as the calculated values from the absorption measurement after treating with RITC. It has been reported that the number of surface silanol $(\equiv \mathrm{Si}-\mathrm{OH})$ 
groups on the silica surface could be measured by various techniques such as IR spectroscopy and inverse reaction chromatography; surface $\mathrm{Si}-\mathrm{OH}$ concentrations vary in the range $3.3 \sim 7 \mathrm{ea} / \mathrm{nm}^{2}$ [26-28]. It can be assumed that one APTMS reacts with three $\mathrm{Si}-\mathrm{OH}$ sites on the surface to give the minimum values for the number of amine sites in the range $1.2 \sim 2.3 \mathrm{ea} / \mathrm{nm}^{2}$, which are comparable to the values obtained from the back titration method in our measurements $\left(2.7 \mathrm{ea} / \mathrm{nm}^{2}\right)$. From the saturation value of $\mathrm{SiO}_{2}$-APTMS(RITC) nanoparticles in Figure 2(a), the number of effective amine sites reacting with RITC can be also calculated as $0.44 \mathrm{ea} / \mathrm{nm}^{2}$ using the calibration curve of free RITC in ethanol solution (see Figure S4, based on the assumption that the absorption coefficient of RITC is not very different when it is attached on the surface). The differences from both analyses can be explained that only one out of six amino $\left(-\mathrm{NH}_{2}\right.$ and $\left.-\mathrm{NH}-\right)$ groups can react with RITC probably due to the steric hindrance from the bulky and flat RITC molecule. Similar quantitative analyses were carried out for $\mathrm{SiO}_{2}$-DETAS and $\mathrm{SiO}_{2}$-DETAS(RITC) nanoparticles, which had three amine sites per DETAS ligand molecule. As expected, the number of amine sites measured by back titration was $7.7 \mathrm{ea} / \mathrm{nm}^{2}$ and that of effective amine sites measured by absorption calibration was $1.3 \mathrm{ea} / \mathrm{nm}^{2}$, almost three times larger than $\mathrm{SiO}_{2}$-APTMS cases. To check the reproducibility and reliability of our quantitative analysis methods, a further chemical reaction was carried out from the amine sites of $\mathrm{SiO}_{2}$-APTMS, and the number of new functional sites was measured. For the quantitative conversion of each generation in the amineterminal dendrimer synthesis, it is known that a terminal primary amine could be successively reacted with methyl acrylate (MA) and ethylenediamine (EDA) to form one tertiary amine and two terminal primary amines (Figure S5) [29]. The same reaction was carried out with $\mathrm{SiO}_{2}-\mathrm{APTMS}$ as illustrated in Scheme 1(b), and the number of amine sites in resulting $\mathrm{SiO}_{2}$-EDA was again measured by back titration. The number of amine sites per $\mathrm{nm}^{2}$ was increased from 2.7 for $\mathrm{SiO}_{2}$-APTMS to 6.8 for $\mathrm{SiO}_{2}$-EDA, as shown in Table 1, corroborating that the conversion reactions proceeded almost quantitatively.

It is well known that the actual number of aminofunctional groups on the modified surfaces is changed with time due to the instability of surface layers. It has been also proposed that some amino group on the surface is bent toward the surface, and the bent amino group is interacted with circumjacent silanol group by hydrogen bonding or acid-base interaction to form ion pairs. Such interactions can reduce the actual reactivity of amine groups [30]. In our surface modification method forming monolayer with amino-functionalized trialkoxysilanes, however, these types of interaction of amine groups with surface silanol groups (so-called backbiting interactions) were reduced significantly and the reactivity of amino group could be maintained for a long period of time without showing any differences in the reactivity. After the surface modification, $\mathrm{SiO}_{2}$-DETAS nanoparticles were stored in ethanol for 1.5 month. Then, $\mathrm{SiO}_{2}$-DETAS was treated with RITC and $\mathrm{SiO}_{2}$-DETAS(RITC) nanoparticles were purified by centrifugation/redispersion processes. The absorption intensities from Rhodamine B units from $\mathrm{SiO}_{2}$-DETAS(RITC) nanoparticles did not show any significant differences even after 1.5 month storage of $\mathrm{SiO}_{2}$-DETAS in ethanol solution, proving the excellent stability of surface aminofunctional groups and the reliable control of surface functionality in our modification method (Figure S6).

\section{Conclusion}

It has been demonstrated that a monolayer modification on the surface of $\mathrm{SiO}_{2}$ nanoparticles can be obtained by using stable ethanol solutions of organofunctional trialkoxysilanes such as APTMS and DETAS. Precise characterizations with transmission electron microscopy (TEM) and dynamic light scattering (DLS) spectrophotometer have confirmed the formation of monolayer modification. The total number of amino groups on the surface of $\mathrm{SiO}_{2}$ nanoparticle were quantitatively analyzed by a simple acid-base back titration method and the effective number of amino groups for the successive chemical reactions was also determined by spectroscopic measurements after treating with fluorescent Rhodamine B isothiocyanate (RITC) molecule, showing well-matched results with known values from literatures. Furthermore, the reproducibility and reliability of our quantitative analysis methods were confirmed by checking the change of amine sites of $\mathrm{SiO}_{2}$-APTMS after the quantitative conversion of the terminal primary amine to one tertiary amine and two terminal primary amines by successive reactions with methyl acrylate (MA) and ethylenediamine (EDA), a process well-developed in dendrimer synthetic methods. We believe that our method to generate monolayer modification and analyze the number of amine sites on nanoparticle surface will be useful in nano-bio research applications, such as sensors, diagnosis, and drug or gene deliveries, where reliable and reproducible modification and quantitative analysis are very critical.

\section{Acknowledgments}

This work was supported by the Technology Development Program for Agriculture and Forestry (TDPAF), Ministry of Agriculture, Forestry, and Fisheries, Republic of Korea (109146-03-2-CG000), and the Nano R\&D Program through the National Research Foundation of Korea (NRF) funded by the Ministry of Education, Science, and Technology (20110019174). H. S. Jung and D.-S. Moon are grateful for the award of a BK21 fellowship.

\section{References}

[1] W. Stöber, A. Fink, and E. Bohn, "Controlled growth of monodisperse silica spheres in the micron size range," Journal of Colloid And Interface Science, vol. 26, no. 1, pp. 62-69, 1968.

[2] H. E. Bergna and W. O. Roberts, Colloidal Silica: Fundamentals and Applications, Taylor and Francis, Boca Raton, Fla, USA, 2005.

[3] E. F. Vansant, P. V. d. Voort, and K. C. Vrancken, Characterization and Chemical Modification of the Silica Surface, Elsevier, New York, NY, USA, 1995. 
[4] W. Tan, K. Wang, X. He et al., "Bionanotechnology based on silica nanoparticles," Medicinal Research Reviews, vol. 24, no. 5, pp. 621-638, 2004.

[5] W. Lin, Y.-W. Huang, X.-D. Zhou, and Y. Ma, "In vitro toxicity of silica nanoparticles in human lung cancer cells," Toxicology and Applied Pharmacology, vol. 217, no. 3, pp. 252-259, 2006.

[6] Y. R. Yeon, Y. J. Park, J.-S. Lee, J.-W. Park, S.-G. Kang, and C.H. Jun, "Sc(OTf $)_{3}$-mediated silylation of hydroxy functional groups on a solid surface: a catalytic grafting method operating at room temperature," Angewandte Chemie, vol. 47, no. 1, pp. 109-112, 2008.

[7] Z. Xu, Q. Zeng, G. Lu, and A. Yu, "Inorganic nanoparticles as carriers for efficient cellular delivery," Chemical Engineering Science, vol. 61, p. 1027, 2006.

[8] P. Watson, A. T. Jones, and D. J. Stephens, "Intracellular trafficking pathways and drug delivery: fluorescence imaging of living and fixed cells," Advanced Drug Delivery Reviews, vol. 57, no. 1, pp. 43-61, 2005.

[9] V. Salgueiriño-Maceira and M. A. Correa-Duarte, "Increasing the complexity of magnetic core/shell structured nanocomposites for biological applications," Advanced Materials, vol. 19, no. 23, pp. 4131-4144, 2007.

[10] V. Salgueiriño-Maceira, M. A. Correa-Duarte, M. Spasova, L. M. Liz-Marzán, and M. Farle, "Composite silica spheres with magnetic and luminescent functionalities," Advanced Functional Materials, vol. 16, no. 4, pp. 509-514, 2006.

[11] A. Guerrero-Martínez, J. Pérez-Juste, and L. M. Liz-Marzán, "Recent progress on silica coating of nanoparticles and related nanomaterials," Advanced Materials, vol. 22, no. 11, pp. 1182$1195,2010$.

[12] C.-H. Chiang, H. Ishida, and J. L. Koenig, "The structure of $\gamma$ aminopropyltriethoxysilane on glass surfaces," Journal of Colloid And Interface Science, vol. 74, no. 2, pp. 396-404, 1980.

[13] D. Knopp, D. Tang, and R. Niessner, "Bioanalytical applications of biomolecule-functionalized nanometer-sized doped silica particles," Analytica Chimica Acta, vol. 647, no. 1, pp. 14 30, 2009.

[14] K. M. R. Kallury, U. J. Krull, and M. Thompson, "X-ray photoelectron spectroscopy of silica surfaces treated with polyfunctional silanes," Analytical Chemistry, vol. 60, no. 2, pp. 169-172, 1988.

[15] G. S. Caravajal, D. E. Leyden, G. R. Quinting, and G. E. Maciel, "Structural characterization of (3-aminopropyl)triethoxysilane-modified silicas by silicon-29 and carbon-13 nuclear magnetic resonance," Analytical Chemistry, vol. 60, no. 17, pp. 1776-1786, 1988.

[16] D. J. Kelly and D. E. Leyden, "Thermodynamic and kinetic investigation of the protonation of silica-immobilized 3aminopropylsilane," Journal of Colloid And Interface Science, vol. 147, no. 1, pp. 213-224, 1991.

[17] K. M. R. Kallury, P. M. Macdonald, and M. Thompson, "Effect of surface water and base catalysis on the silanization of silica by (aminopropyl)alkoxysilanes studied by X-ray photoelectron spectroscopy and 13C cross-polarization/magic angle spinning nuclear magnetic resonance," Langmuir, vol. 10, no. 2, pp. 492-499, 1994.

[18] K. C. Vrancken, K. Possemiers, P. Van Der Voort, and E. F. Vansant, "Surface modification of silica gels with aminoorganosilanes," Colloids and Surfaces A, vol. 98, no. 3, pp. 235-241, 1995.

[19] S. R. Corrie, G. A. Lawrie, and M. Trau, "Quantitative analysis and characterization of biofunctionalized fluorescent silica particles," Langmuir, vol. 22, no. 6, pp. 2731-2737, 2006.
[20] X. Zhao and R. Kopelman, "Mechanism of organosilane selfassembled monolayer formation on silica studied by secondharmonic generation," Journal of Physical Chemistry, vol. 100, no. 26, pp. 11014-11018, 1996.

[21] L. Jin, A. Horgan, and R. Levicky, "Preparation of end-tethered DNA monolayers on siliceous surfaces using heterobifunctional cross-linkers," Langmuir, vol. 19, no. 17, pp. 6968-6975, 2003.

[22] J. A. Howarter and J. P. Youngblood, "Optimization of silica silanization by 3-aminopropyltriethoxysilane," Langmuir, vol. 22, no. 26, pp. 11142-11147, 2006.

[23] E. T. Vandenberg, L. Bertilsson, B. Liedberg et al., "Structure of 3-aminopropyl triethoxy silane on silicon oxide," Journal of Colloid And Interface Science, vol. 147, no. 1, pp. 103-118, 1991.

[24] G. T. Hermanson, Bioconjugate Techniques, Academic Press, San Diego, Calif, USA, 2nd edition, 1996.

[25] D. A. Skoog and D. M. West, Analytical Chemistry, Saunders College, Philadelphia, Pa, USA, 3rd edition, 1980.

[26] V. Y. Davydov, A. V. Kiselev, and L. T. Zhuravlev, "Study of the surface and bulk hydroxyl groups of silica by infra-red spectra and $\mathrm{D}_{2} \mathrm{O}$-exchange," Transactions of the Faraday Society, vol. 60, pp. 2254-2264, 1964.

[27] V. A. Bakaev and C. G. Pantano, "Inverse reaction chromatography. 2. hydrogen/deuterium exchange with silanol groups on the surface of fumed silica," Journal of Physical Chemistry C, vol. 113, no. 31, pp. 13894-13898, 2009.

[28] R. K. Iler, The Chemistry of Silica, Jon Wiley \& Sons, New York, NY, USA, 1979.

[29] K. Sakai, M. Kunitake, T. C. Teng et al., "Designable size exclusion chromatography columns based on dendritic polymermodified porous silica particles," Chemistry of Materials, vol. 15, no. 21, pp. 4091-4097, 2003.

[30] N. K. Kamisetty, S. P. Pack, M. Nonogawa, K. C. Devarayapalli, T. Kodaki, and K. Makino, "Development of an efficient amine-functionalized glass platform by additional silanization treatment with alkylsilane," Analytical and Bioanalytical Chemistry, vol. 386, no. 6, pp. 1649-1655, 2006. 

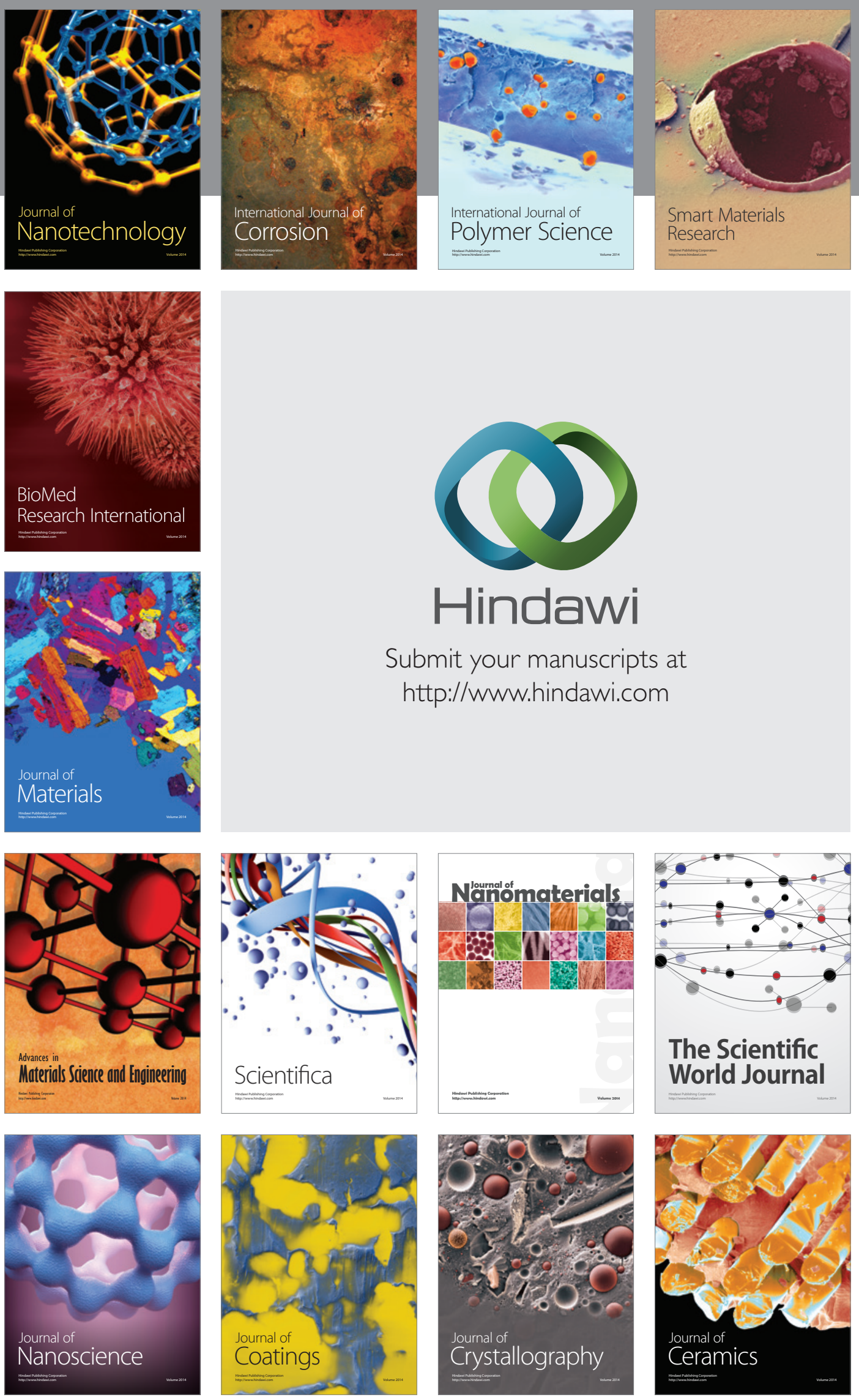

The Scientific World Journal

Submit your manuscripts at

http://www.hindawi.com

\section{World Journal}

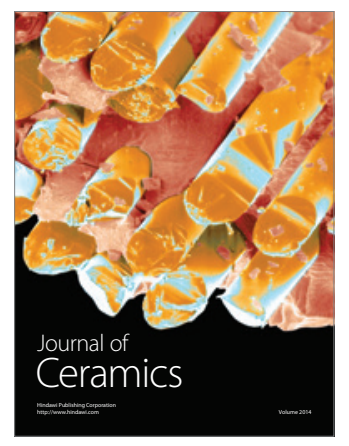

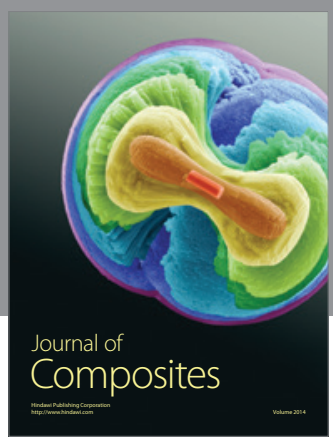
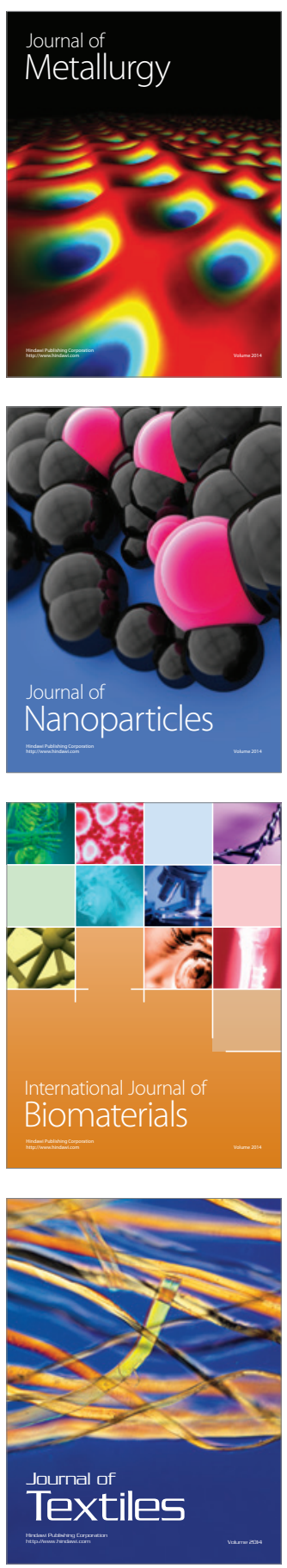